\section{$\underset{\substack{\text { hommes } \\ \text { \& migrations }}}{ }$}

\section{Hommes \& migrations}

Revue française de référence sur les dynamiques

migratoires

1302 | 2013

Le Japon, pays d'immigration?

\title{
Madagascar, musiques foisonnantes
}

\section{François Bensignor}

\section{CpenEdition \\ Journals}

\section{Édition électronique}

URL : http://journals.openedition.org/hommesmigrations/2521

DOI : 10.4000/hommesmigrations.2521

ISSN : 2262-3353

\section{Éditeur}

Musée national de l'histoire de l'immigration

\section{Édition imprimée}

Date de publication : 1 avril 2013

Pagination : 176-179

ISBN : 978-2-919040-22-3

ISSN : $1142-852 X$

\section{Référence électronique}

François Bensignor, « Madagascar, musiques foisonnantes », Hommes \& migrations [En ligne], 1302 |

2013, mis en ligne le 16 septembre 2013, consulté le 22 septembre 2020. URL : http://

journals.openedition.org/hommesmigrations/2521; DOI : https://doi.org/10.4000/

hommesmigrations. 2521 


\title{
MADAGASCAR, MUSIQUES FOISONNANTES
}

\author{
FRANÇOIS BENSIGNOR
}

es 18 tribus peuplant Madagascar partagent Lune façon bien à elles d'envisager la musique. Elles s'appuient sur deux constantes : un art consommé du chant polyphonique, un mélange de rythmes ternaires et binaires structurant mélodies et paroles. Sur cette base, invention, fantaisie, génie de l'harmonie et de la mélodie stimulent l'émulation entre des musiciens fiers de développer l'originalité propre à leurs cultures respectives. Petite revue de cette grande diversité.

Lîle de Madagascar, surnommée "l'île rouge" à cause de la couleur de la latérite qui en constitue la terre, est un petit continent à elle seule. D'innombrables espèces de plantes et d'animaux s'y développent depuis des temps reculés. Mais il semble que l'homo sapiens n'ait abordé ses rivages que vers les débuts de l'ère chrétienne. Les chercheurs montrent que les premiers humains ayant fait souche sur la Grande île furent des navigateurs austronésiens. Ceux-là mêmes que les pirogues à balanciers ont portés dans toute l'Indonésie, sur l'étendue du Pacifique vers les archipels micronésiens, mélanésiens, polynésiens et jusqu'en mer de Tasmanie. La langue malgache, qui unifie les 18 tribus de l'île, révèle des similitudes avec certaines langues de Bornéo. Et l'on retrouve des cithares tubulaires, semblables à l'emblématique valiha de Madagascar, en Malaisie et aux Philippines, comme sur les hauts plateaux du Cambodge et du Vietnam.

La population malgache, quicompte plus de 21 millions d'habitants, est un mélange de multiples apports. Ceux provenant d'Indonésie et d'Afrique sont essentiels. Mais la culture insulaire a également été marquée par les populations qui ont débarqué dans l'île au cours des siècles : Arabes, Persans et autres peuples islamisés, Indiens, Européens, Chinois, etc. Ce brassage a constitué "un peuple intermédiaire guère facile à déterminer et pourtant typiquement reconnaissable: le Malgache contemporain", écrit le poète Jacques Rabemananjara.

Les Malgaches ont beau être unis par une culture et une langue communes, ils se divisent selon des groupes ethniques établis dans les diverses régions de la Grande île. Coiffures, vêtements, architectures, langues ou accents spécifiques sont autant d'éléments marquant leurs différences. Évoquons les principaux. Les Mérina ("ceux qui habitent les hauteurs"), descendants des peuples asiatiques de Madagascar, vivent sur les hautes terres du centre autour de la capitale Antananarivo. Les Betsileo ("ceux qui sont invincibles") occupent la province de Fianarantsoa au CentreEst. Les Betsimisaraka ("ceux qui ne se séparent pas") se répartissent sur la côte orientale. Tout le long de la côte occidentale se trouvent les Sakalava. À l'extrême-sud, les Antandroy vivent sur les terres arides où poussent les épineux. Leurs voisins à l'ouest sont les Mahafaly et sur la côte sud, les Vezo, descendants de pêcheurs d'Afrique orientale. Sur la côte sud-est, les Antesaka et les Atemoro sont les héritiers des populations arabes. Au sud du plateau central, les Bara 

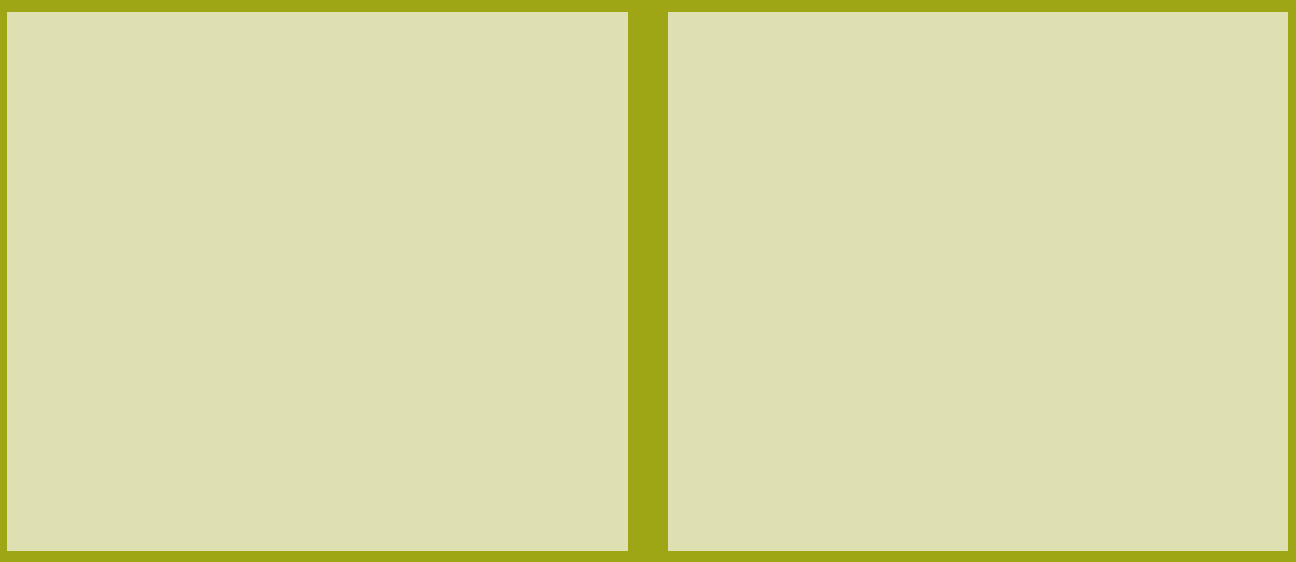

sont des éleveurs de bœufs, qu'ils se glorifient de se voler mutuellement...

On est frappé par l'appétence et l'aptitude musicales des Malgaches. Femme ou homme, riche ou pauvre, bouvier des hauts plateaux ou chauffeur de taxi dans la pollution de la capitale, tout un chacun sait naturellement tenir la cadence 12/8 omniprésente. La faculté de construire des instruments aux sons étonnants à partir des matériaux les plus usuels paraît aussi chez eux comme une seconde nature. Quant aux harmonisations subtiles de la polyphonie vocale, elles semblent à la portée de toutes les sociétés traditionnelles, qui entretiennent de fortes solidarités humaines. Il faut sans doute préciser que, dans la tradition, un chanteur ne chante en solitaire que lorsqu'il garde les zébus. Le chant choral, hérité des fêtes rituelles, imprègne toutes formes d'expression vocale à Madagascar. Des solistes émergent, chantent des couplets ou improvisent, mais ils sont toujours soutenus par le chœur.

\section{La musique au cœur de la Grande Île}

La mythologietémoignedu rôlefondamental,voire fondateur, de la musique dans toutes les tribus de l'île. Pour les Tsimihety du Nord-Ouest, c'est le dieu créateur, Zanahary lui-même, qui fit don des instruments de musique aux hommes et aux femmes, afin qu'ils puissent se divertir. Le nom du roi qui régna sur les hauts plateaux au XIII e siècle, et auquel remontent les généalogies de toutes les dynasties royales malgaches, est Andrianampongadanitra, qui signifie : le "Prince des tambours célestes". Les trois principaux royaumes constitués dès le $X V l^{e}$ siècle ont développé, en même temps que leurs costumes et parures spécifiques, des styles musicaux bien à eux.

Les Betsileo avaient autrefois une spécialité de chant épique, interprété par des ménestrels, réputés également pour leurs prouesses poétiques. Très prisé aujourd'hui, le "rija", chant polyphonique typique de l'art vocal des Betsileo accompagné de battements de mains, traduit la joie populaire, parfois même jusqu'au déchaînement. L'influence chrétienne a tempéré ce genre choral dans une forme plus récente, le "rafindraony", évoquant les valeurs morales de la société.

Les Sakalava attribuent des pouvoirs magiques aux instruments de musiques. Ils affectionnent le tempo moyen du "jijy", ses paroles tour à tour moqueuses ou louangeuses, ses thèmes narratifs ou subversifs. Lointaines descendantes de populations arabisées, leurs cérémonies religieuses s'accompagnent de chants, de danses et de battements de mains, de tambours et de valiha. Installé au sud-ouest de l'île dès le XVIe siècle, le royaume Sakalava connut son apogée au XVIIe, contrôlant 


\section{MUSIQUES}

toute la côte ouest de Madagascar. Mais, avec la montée du royaume Merina au XVIII ${ }^{e}$ siècle, son influence commença à décliner.

Jusqu'au XVIII siècle, les rois Merina ont noué des alliances avec les autres royaumes à travers les mariages et le commerce de zébus ou d'esclaves. Mais lorsque les Anglais font au roi le cadeau de fusils, les Merina partent en guerre, étendant un pouvoir qu'ils vont exercer jusqu'à l'arrivée des colonisateurs français en 1896. Leur musique se distingue par une profusion

cratisée et répandue dans toutes les régions, devenant un symbole de Madagascar. Traditionnellement, les cordes étaient faites avec les longs fils d'écorce détachés du bambou et tendus sur des frettes amovibles permettant l'accordage. Le plus souvent au nombre de 16 (parfois 14, 18, 20 ou 24), ces cordes végétales sont aujourd'hui remplacées par des cordes métalliques. La valiha accompagnait autrefois les cérémonies religieuses et les rituels de possession comme la "tromba" et le "bilo", ainsi que le fameux rite de retournement des morts "famadihana".

À cause de sa faible portée sonore, elle a été remplacée d'abord par la petite flûte "sodina", puis par l'accordéon. Arrivé avec les Occidentaux d'abord sous sa forme diatonique, cet instrument est aujourd'hui partie intégrante de la tradition malgache.

Afin de l'adapter à leurs harmonies, les musiciens en rognent les sifflets, opération qu'ils appellent "circoncire" l'accordéon, qui prend alors le nom de "gorodo".

\section{Vivacité de la musique traditionnelle malgache}

Avec la colonisation, le gorodo entre parmi les instruments des grands orchestresaccompagnantle"hira-gasy" (littéralement "chants malgaches"). Forme d'opéra ou théâtre chanté, la tradition du hira-gasy remonte au

de styles et de pratiques d'une grande richesse. De tous leurs instruments, la valiha est celui qui fascine les voyageurs depuis plus de cinq siècles. Autrefois l'apanage des princes, qui laissaient pousser leurs ongles afin d'en jouer, cette cithare tubulaire faite à partir d'un bambou s'est démo-
$\mathrm{XV}^{\mathrm{e}}$ siècle. De grands rois Merina l'utilisèrent pour mobiliser et éduquer le peuple. Spectacle complet comprenant l'art oratoire du "kabary", des chants, de la musique et de la danse, le hiragasy se déroule sur une journée entière selon une structure précise. Au temps de la colonisa- 


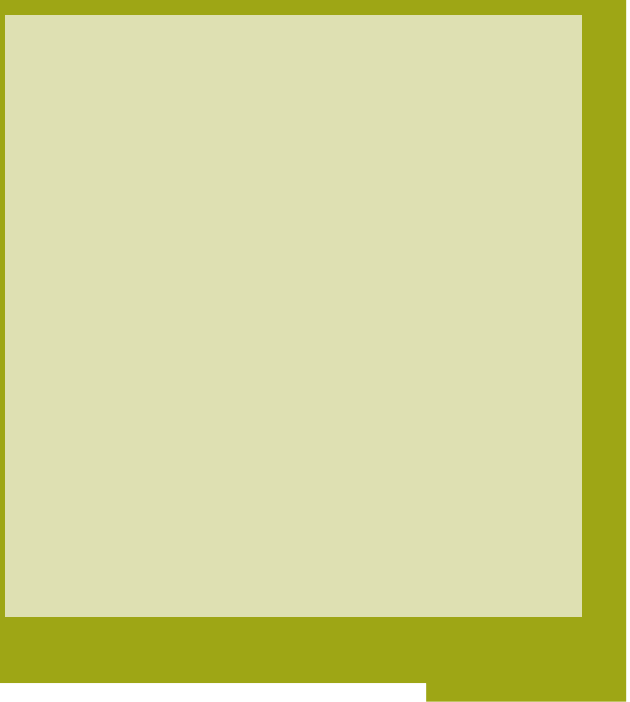

tion, les habitants des hauts plateaux, réputés pour nouer et dénouer les jeux de la politique, utilisèrent le hira-gasy sur un mode satirique, très critique envers l'administration. Les paroles, que les Européens entendaient sans comprendre, permettaient aux Malgaches de communiquer entre eux et de rire aux dépens de l'oppresseur. Si la forme complète du spectacle tel qu'il se jouait à l'apogée du genre (XVIII et XIXe siècles) a presque disparu, le répertoire chanté "ba-gasy" en conserve le mode d'expression dramaturgique ainsi que les thèmes principaux, qui traitent de l'amitié et de l'entraide.

Alors qu'il était menacé par les influences modernes, ce genre a connu une véritable renaissance dans les années 1990 grâce à la formation du groupe Feo-Gasy, initiée par Erick Manana. Les paroles des chansons relèvent d'un art poétique oral qui fonctionne sur le mode question-réponse et conserve une certaine théâtralité. Mais l'esprit ba-gasy proprement dit réside avant tout dans la façon de chanter et notamment dans la valeur donnée à chaque mot. Avec ses paroles d'amour, ses vers mélancoliques ou ses couplets humo- ristiques, la chanson traditionnelle malgache est surtout faite de morales chantées. On racontera l'histoire de ce jeune homme, parti sans tenir compte des conseils de ses parents, qui a mal tourné, puis est revenu penaud.

Ou celle de cet ivrogne, de ce coq de village... On chantera la femme, la beauté de son corps qui danse. On fera l'éloge d'un ancêtre. Souvent on chantera une prière pour l'adresser à Dieu et aux ancêtres, un héritage des rituels anciens, particulièrement dans les régions côtières. Il y a toujours un fond mystique chez le Malgache, qui croit en la force supérieure de Zanahary, le dieu qui est au-dessus de tout.

\section{Discographie :}

- Madagascar - Antandroy ; Antanosy ; Bara ; Betsileo; Betsimisaraka ; Mahafaly; Merina ; Sakalava (Prophet)

- Madagascar - Anthologie des Voix Antandroy, Antanosy, Bara, Betsileo, Boina, Mahafaly, Merina, Mikea et Menabe Sakalava (Inédit)

- Madagascar-Musiques de la côte et des hauts plateaux, 1929-1931 (Frémeaux \& Associés)

- Madagascar-Musique d'évocation des Esprits, région de Tamatave (Unesco)

- Madagascar - Musique des Sakalava Menabe. Hommage à Mama Sana (Inédit)

- Madagascar-Musique paysanne Betsileo Lalangina (Buda Musique)

- Madagascar - Pays Antandroy (Ocora Radio France)

- Madagascar - Pays Betsimisaraka (Unesco)

- Madagascar - Pays Mikea (Ocora Radio France)

- Madagascar-Tsapiky, panorama d'une jeune musique de Tuléar (Arion)

- Musiques de Madagascar (Buda Musique)

- Tulear Never Sleeps - Tsapiky Guitars from South West Madagascar (Earthworks/Stern's) 


\section{NY MALAGASY ORKESTRA}

"Orchestre traditionnel de création", comme il s'intitule lui-même, le Ny Malagasy Orkestra a été formé afin de présenter le foisonnement des styles qui continuent de vivre à Madagascar. Initiateur du projet, Justin Vali, célèbre joueur de valiha résidant en France et Grand Prix de la Sacem musiques traditionnelles en 2006, profite d'une de ses tournées sur les routes poussiéreuses de la Grande île pour sélectionner certains des musiciens les plus réputés et les plus créatifs des régions qu'il traverse.

À chaque étape de la tournée - Tuléar, Nosy-Be, Fianarantsoa, Tamatave, etc. - il prend soin, avec Tao Ravao, musicien et directeur artistique de la future formation, d'organiser une journée consacrée à rencontrer des musiciens et à jouer avec eux. II ne s'agit pas d'auditions à l'européenne, mais de "bœufs", au cours desquels émergent les personnalités musicales les plus représentatives de leurs cultures. Les quatorze musiciens retenus sont des maitres dans la pratique de leurs instruments et des compositeurs reconnus par leurs pairs dans leurs régions respectives.

Tous se retrouvent à Antananarivo et le groupe est formé avec une dizaine d'entre eux. Sur une période d'un an, ils vont travailler à l'élaboration du répertoire. Le disque Masoala, produit par le musicien et historien de la musique Thierry Bongarts Lebbe, est sorti en 2010. Chaque musicien apporte au moins deux ou trois de ses compositions et le choix des pièces les plus éloquentes est fait de manière collégiale. Très loin du "catalogue touristique" le plus souvent présenté par les maisons de disques occidentales, l'album comme le spectacle proposent un voyage au cœur de l'identité culturelle de chaque ethnie malgache.
Justin Vali, le fondateur du groupe, tire de sa valiha une musique à la fraîcheur limpide, héritée des grands maîtres Sylvestre Randafison et Rakotozafy, qui initièrent son raffinement contemporain. L'accordéoniste, violoniste et chanteur Jean-Donné Ramanananerisoa, issu d'une grande lignée de musiciens, incarne la permanence du genre hira gasy. Tout comme Dieudonné Randriamanantena, poly-instrumentiste et chanteur, directeur d'une troupe réputée d'Antananarivo. Ce dernier est un maître incontesté du "vakodranzana", forme de folklore traditionnel mis en scène et intégrant diverses traditions rurales.

L'accordéoniste Jean-Piso Rebily, doyen du groupe, et son benjamin Chrysanthe Velomijoro, guitariste soliste, sont originaires de la Province de Tuléar. Comme le climat du Sud, la musique y est torride, aiguillonnée par le tempo rapide du "tsapiky", dont Jean-Piso est un grand spécialiste. Cette vélocité anime les doigts de Chrysanthe, virtuose à la finesse d'un D'Gary. Remanindry vient du pays des Antandroy, la région des épines, également dans la province de Tuléar.

Violoniste, maître du style "beko", ce berger de profession, qui tire des sons magiques de sa viole "lokanga", joue également un rôle social important dans sa communauté, où il règle les conflits de personnes. Gilberto Moravelo, chanteur et virtuose de la mandoline originaire du Sud, est un maître des styles "pitiky langay" et "sarandra". Le style chanté de Fort-Dauphin (Tolanaro) n'a pas de secrets pour lui.

Maurice Razanakoto et Francis "Fafa" Rakotoson Andrianomanana jouent la douce musique des Betsileo des hauts plateaux du Centre. Charpentier et poète, Maurice a modernisé le "kabossy", petite guitare des gardiens de zébus, en lui 
confectionnant deux manches. Quant à Fafa, il joue du "jejo voatavo", cithare sur bâton munie d'une calebasse résonateur unique à Madagascar chez les Betsileo. Elle a dû voyager sur les bateaux venus d'Asie : une cithare similaire est jouée chez les montagnards des hauts plateaux du Vietnam. Tiana Ramarokoto, bon chanteur et musicien de studio, est à l'aise sur tous les instruments. Ses connaissances en lutherie transcendent les traditions. Quant à son expérience des tournées internationales, elle fait de lui l'élément de toutes les synthèses réunies dans la musique du Ny Malagasy Orkestra. Cette formation exceptionnelle est en tournée en France en juillet 2013. Elle reviendra en novembre 2014 pour une résidence de création avec Emmanuel Monnet et les musiciens du département Musique traditionnelle du conservatoire de Montluçon. Cette création devrait associer des instruments et répertoires des traditions françaises (accordéon, vièle à roue, cabrette, etc.) et malgaches.

\section{Discographie :}

- Ny Malagasy Orkestra, Masoala Cing Planètes / l'Autre distribution, 2010)

\section{Tournée 2013}

\section{Ny Malagasy Orkestra :}

30 juin : Les Orientales de Saint-Florent-leVieil (49); 6 juillet : Châtellerault (86); 7 juillet: Paris (75), Arènes de Montmartre ; 10 juillet: Tournon (07) ; 12 juillet:

Horizonte Festival, Coblence (Allemagne); 14 juillet : Châlons-en-Champagne (51) ; 15 juillet : Albertville (73), 16 juillet : La Rochette (73) ; 19 juillet : Vertheuil (33) ; 20 juillet : festival Résonances, Rochefort (17) ; 23 juillet : Orgelet (39) ; 24 juillet : Chambon-sur-Voueize (23) ; 26 juillet : Bourges (18) ; 28 juillet : Pontigny (89). 